\title{
Subacute Infective Endocarditis Secondary to Cardiobacterium hominis in a Patient with Mitral Valve Prolapse
}

Lakshman Vasanthamohan MD, Nadine Kronfli MPH MD FRCP(C), Chenchen Hou MD, Ally P.H. Prebtani BScPhm MD ABIM FRCP(C), Anjali Shroff MD FRCPC

\begin{abstract}
About the Authors
Lakshman Vasanthamohan, is PGY-2 in the Department of Internal Medicine at McMaster University, Hamilton, ON. Nadine Kronfli is PGY-5 in the Division of Infectious Diseases at McMaster University, Hamilton, ON. Chenchen Hou is PGY-3 in the Department of Internal Medicine at McMaster University, Hamilton, ON. Ally P.H. Prebtani is professor of Medicine, Division of Endocrinology and Metabolism at McMaster University, Hamilton, ON. Anjali Shroff is assistant professor, Division of Infectious Diseases Metabolism at McMaster University, Hamilton, ON.
\end{abstract}

Submitted: January 16, 2017. Accepted: August 2, 2017. Published November 7, 2017

\begin{abstract}
We present the case of a 42-year-old man with a history of mitral valve prolapse (MVP) presenting with subacute native valve endocarditis secondary to Cardiobacterium hominis infection. He presented with non-specific symptoms of generalized fatigue and malaise subsequent to a complication-free dental cleaning. He was treated initially with 4 weeks of ceftriaxone, but ultimately required mitral valve replacement due to severe valvular degeneration. This case provides a review of the pathophysiology and presentations of infective endocarditis (IE) secondary to HACEK organisms. We will review risk factors, as well as the evidence and current guidelines for pre-procedural IE prophylaxis.
\end{abstract}

\section{Résumé}

Il s'agit du cas d'un homme âgé de 42 ans ayant des antécédents de prolapsus valvulaire mitral (PVM) lié à une endocardite sur valve native subaiguë attribuable à une infection causée par Cardiobacterium hominis. Le patient présente des symptômes non-spécifiques de fatigue générale et de malaise à la suite d'un nettoyage dentaire sétant déroulé sans complications. Après 4 semaines de traitement à la ceftriaxone, il a finalement dû subir le remplacement de la valvule mitrale en raison d'une grave dégradation valvulaire. L'étude de ce cas fournit une rétrospective de la physiopathologie et des différentes présentations d'une endocardite infectieuse (EI) secondaire aux organismes HACEK. Nous passons en revue différents facteurs de risque, ainsi que les données probantes et les lignes directrices actuelles concernant les mesures préventives de prophylaxie relative à l'EI.

\section{Case Presentation}

A 42-year old man with a history of mitral valve prolapse (MVP) presented to the emergency department in August 2015 following direct referral by his cardiologist. A transthoracic echocardiogram (TTE) showed thickening of his posterior mitral valve leaflet, suspicious for a vegetation. He endorsed a history of worsening generalized fatigue and malaise over months that were beginning to impair his day to day function, but otherwise, a review of systems was negative. Of note, he denied any history of fevers, chills, night sweats or weight loss, and had no shortness of breath or chest pain. Four months prior to the onset of his symptoms, he had a complication-free dental cleaning, but no other procedures. As per AHA/IDSA guidelines, he did not take any antimicrobial prophylaxis prior 
to his cleaning. His past medical history was unremarkable with no history of autoimmune disease, intravenous drug use or chronic indwelling devices such as catheters or lines. He was not taking any prescribed or over the counter medications and had no known allergies. On social history, there was no recent travel, he was a non-smoker, minimal alcohol drinker, and his family owned a dog, a gecko, and a new kitten that was a few weeks old and had recently given him a superficial bite on the arm that had not broken through his skin.

On examination, he looked systemically well. His vital signs were stable and he was afebrile. Precordial exam was normal except for the presence of a grade III/VI holosystolic murmur best heard at the apex, radiating to the axilla. There was no evidence of congestive heart failure. There were no stigmata of infective endocarditis (IE). The remainder of the exam was unremarkable.

Pertinent laboratory investigations included a white blood cell count of 13.4 (normal range 4.0-11.0 $\times 109 / \mathrm{L}$ ) with an absolute neutrophil count of 11.4 (normal range $2.0-7.5 \times 109 / \mathrm{L}$ ) and an elevated C-reactive protein at 28.6 (normal $<5 \mathrm{mg} / \mathrm{L})$. ESR was not ordered. The remainder of his bloodwork and a urinalysis was within normal limits. Serial ECGs showed transient sinus tachycardia without any signs of heart block. A chest radiograph showed no visible abnormalities. Three sequential sets of blood cultures were drawn. A transesophageal echocardiogram (TEE) revealed severe mitral regurgitation as well as thickening and possible myxomatous degeneration of the mitral valve (Figure 1). The presence of a vegetation on the mitral valve could not be ruled out.

Two days following the patient's admission, two sets of blood cultures grew Gram-negative bacilli after 43.2 hours. The long incubation time was inconsistent with most Gram-negative organisms, and given the microbiology lab's comment on the inconsistency of the Gram stain with areas of under-colourization,

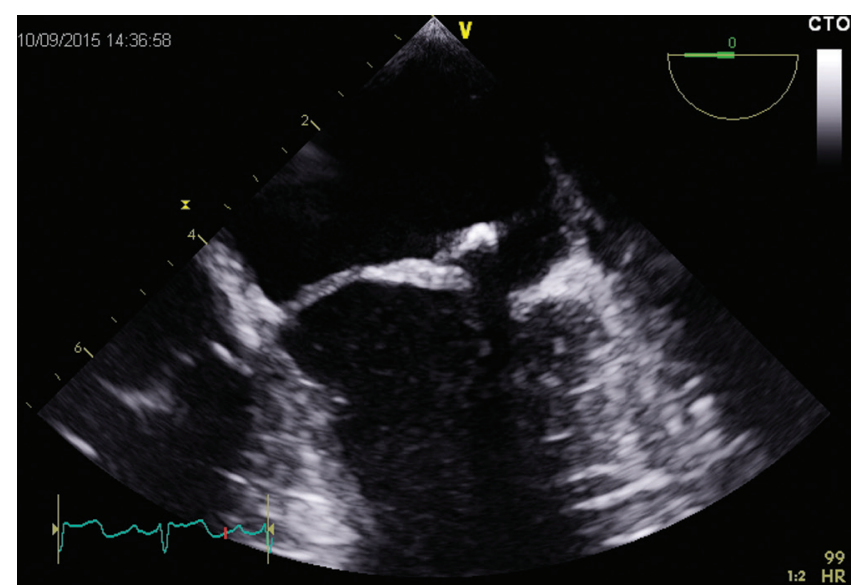

Figure 1. Transesophageal echocardiogram showing a lesion suspicious for a vegetation on the anterior leaflet of the mitral valve. the patient was treated with vancomycin and ceftazidime for broad spectrum coverage. The following day, Cardiobacterium hominis was isolated. The strain was susceptible to beta-lactams. The patient remained clinically stable and was discharged with a PICC line and a 4 -week course of ceftriaxone for C. hominis infective endocarditis (IE). Blood cultures drawn a day after the initiation of treatment were negative. The patient was provided with antimicrobial prophylaxis for future appropriate procedures.

The patient began to experience symptoms of shortness of breath on exertion and palpitations in the months after his initial diagnosis. Repeat echocardiography continued to demonstrate severe degeneration and regurgitation of the mitral valve. His progressive symptoms prompted a referral to cardiac surgery and he underwent a mitral valve replacement in March 2016. Pathology from the surgical tissue sample showed chronic inflammation, fibrosis and myxomatous degeneration but was culture negative several months after his course of antimicrobials.

\section{Discussion}

\section{The HACEK Group}

IE has a diverse pathophysiology and is caused by a variety of mechanisms. The most common etiology is secondary to bacterial infection. Streptococcus and Staphylococcus species are responsible for approximately $70-80 \%$ of cases of IE (Table 1). A group of fastidious Gram-negative organisms referred to as the HACEK organisms (Haemophilus parainfluenzae, Aggregatibacter species, Cardiobacterium species, Eikenella corrodens and Kingella kingae) are well-established, albeit infrequent causes of IE (1-3\%). There have been recent changes to the nomenclature as certain several members of the Haemophilus species have been reclassified as Aggregatibacter species. ${ }^{1}$ Distinguishing features of HACEK IE, as identified in a large database review, include a younger patient population and a weaker association with health care associated infections. ${ }^{2}$ Major causes of IE are outlined in Table 1.,4

Table 1. Major Causes of Infective Endocarditis

\begin{tabular}{|l|c|}
\hline \multicolumn{1}{|c|}{ Microorganism } & Approximate proportion of cases \\
\hline Streptococcus species & $38 \%$ \\
\hline Staphylococcus species & $36 \%$ \\
\hline Enterococcus species & $11 \%$ \\
\hline HACEK & $1-3 \%$ \\
\hline Other & $5-7 \%$ \\
\hline Culture negative & $5-7 \%$ \\
\hline
\end{tabular}


The long incubation time required to culture HACEK organisms has been well-documented, almost exclusively taking in excess of 2 days. Previous studies suggested that long incubation times of at least five to seven days were required to culture C. hominis; however, recently published cases and reviews have demonstrated consistent growth within five days. ${ }^{5}$ Similarly, a review of laboratory incubations showed that all cases of HACEK IE were diagnosed within 5 days, and all incubations beyond 5 days yielded no growth. ${ }^{6}$ This is important given the ever-increasing demands on microbiology labs and the extra costs incurred from prolonged incubation with no evident benefit. Current IDSA guidelines recommend three sets (including $8-10 \mathrm{ml}$ of blood each into both an aerobic and anaerobic culture bottles) of sequential blood cultures be drawn to increase the diagnostic yield when investigating for endocarditis. ${ }^{7}$ Our patient's strain of C. hominis was susceptible to beta-lactams, as are approximately $93 \%$ of reported cases. ${ }^{8}$

\section{Cardiobacterium hominis}

This case highlights a few classic features of C. hominis IE. C. hominis is a Gram-negative bacterium which commonly colonizes the oropharynx. Of note, the bacteria are pleomorphic rods and parts of the cell may actually stain Gram-positive. ${ }^{9} \mathrm{~A}$ retrospective study of HACEK IE showed that $C$. hominis had a predominance for late disease presentation with the majority of reported cases (10/12) occurring three or more months following symptom onset. ${ }^{3}$ The common symptoms are constitutional, including fever, chills, fatigue and weight loss. Symptoms of congestive heart failure are more common in cases of $C$. hominis than other HACEK organisms, and approximately $40 \%$ of cases ultimately require surgery on the affected valve. ${ }^{8}$

\section{Risk Factors for C. hominis IE:}

Established risk factors for IE caused by HACEK organisms, and specifically C. hominis, include structural cardiac abnormalities as well as dental procedures, which is understandable given that the bacteria are oral commensals. However, in contrast to the Gram-positive bacteria commonly causing IE, intravenous drug use does not seem to be a risk factor for $C$. hominis IE likely due to its absence on the skin at points of vascular injury. ${ }^{5}$ Dental manipulation has been classically associated with the development of transient bacteremia even in immunocompetent hosts. ${ }^{10}$ More recently, even mild dental manipulation from brushing has been shown to cause transient bacteremia. ${ }^{11}$ While transient bacteremia is typically innocuous, it serves as a risk factor for IE particularly in the setting of structural cardiac abnormalities.

There are several cardiac structural lesions that predispose a patient to bacterial seeding leading to IE. Population-based studies in North America have shown that MVP has superseded rheumatic heart disease as the most common predisposing cardiac condition. ${ }^{11}$ As well in patients with IE and no prior cardiac diagnosis, mitral valve prolapse with or without valvular regurgitation is the most commonly diagnosed underlying cardiac structural abnormality on echocardiography. ${ }^{12}$ The pathophysiology of regurgitant valvular disease predisposing to IE involves turbulent flow through the valve leading to endothelial damage and the development of a vegetation through the aggregation of platelets, fibrin, and clotting factors at the site of the exposed endothelium.

\section{Antibiotic Prophylaxis for IE Prevention}

The utility of antibiotic prophylaxis prior to dental procedures for the prevention of infective endocarditis in patients with MVP has long been debated. Current major guidelines do not recommend prophylaxis for patients with MVP based on the lack of robust evidence for the effectiveness of prophylactic antibiotics in preventing endocarditis. ${ }^{11,13}$ A Cochrane review of pre-procedural antibiotic prophylaxis, updated in 2013, only found one study suitable for inclusion in their analysis: a Dutch case-control study published in $1992 .{ }^{14}$ There was a non-significant increase in the risk of developing IE 180 days post procedure for patients who did not receive antibiotic prophylaxis compared to those who did (odds ratio 1.62 with a $95 \%$ CI of $0.57-4.57$ ). ${ }^{14,15}$ To date, there have not been any randomized controlled trials examining the use of antibiotic prophylaxis in the prevention of IE. Such a study would be challenging to undertake as relatively low event rates would require large numbers of enrolled patients to ensure adequate power. It is clear that this is an area that would benefit from further study in order to further our understanding about the prevention of IE.

Based on the lack of strong evidence supporting antimicrobial prophylaxis, the United Kingdom's 2008 NICE guidelines for IE recommended no prophylaxis ahead of dental surgery for any indication. A subsequent temporal cohort study of the years before and after this guideline showed both a decrease in prescriptions for antimicrobial prophylaxis, as well as an increase in the incidence of IE after the guideline change. ${ }^{16}$ Although this result does not suggest causation, it prompted an amendment to the NICE guidelines in 2016 to recommend against routine antibiotic prophylaxis, but to consider a case-by-case approach. ${ }^{17}$ In comparison, the AHA/IDSA guidelines recommend prophylaxis only to patients at high risk of developing IE (Table 2). ${ }^{11}$ Although the utility and efficacy of prophylaxis is still somewhat unclear, studies have shown that modern methods of prophylaxis with amoxicillin are safe and well-tolerated. ${ }^{18}$ While our patient would not have previously met the 2007 AHA/IDSA criteria for pre-procedural prophylaxis prior to his dental cleaning, his 
episode of endocarditis would qualify him for future prophylaxis with amoxicillin.

Table 2. Cardiac Conditions for which Pre-procedural Antibiotics are Recommended to Prevent IE as per the AHA/IDSA Guidelines

Conditions for which IE Prophylaxis is Recommended

Prosthetic heart valve (mechanical or tissue)

Previous history of IE

Valvulopathy in cardiac transplant patients

Unrepaired cyanotic congenital heart disease

Repaired congenital heart disease with residual defects and in the first 6 months post procedure

\section{Conclusion}

In summary, our case of native valve $C$. hominis IE represents a classic presentation of an uncommonly-occurring disease process. The case emphasizes the importance of a thorough history, physical exam, and laboratory investigations in the workup of potential IE. In patients presenting with fever of unknown origin or new-onset heart failure one should not neglect to ask about major risk factors for IE such as cardiac structural disease and IV drug use, but also enquire about other routes of bacterial exposure including dental procedures and ownership of pets. A review of the literature shows that there is minimal evidence regarding the efficacy of antibiotic prophylaxis prior to dental procedures for all-comers at risk of IE. Further research should focus on specific populations in which procedural prophylaxis would be effective.

\section{References}

1. Janda WM. Update on the HACEK group of fastidious gram-negative bacilli, Part II. Clin Microbiol Newsl 2013;35:95-101. doi:10.1016/j. clinmicnews.2013.05.004.

2. Chambers ST, Murdoch D, Morris A, et al. HACEK infective endocarditis: Characteristics and outcomes from a large, multi-national cohort. PLoS One 2013;8:1-9. doi:10.1371/journal.pone.0063181.
3. Das M, Badley AD, Cockerill FR, et al. Infective endocarditis caused by HACEK microorganisms. Annu Rev Med 1997;48:25-33. doi:10.1146/ annurev.med.48.1.25.

4. Selton-Suty C, Célard M, Le Moing V, et al. Preeminence of staphylococcus aureus in infective endocarditis: A 1-year population-based survey. Clin Infect Dis 2012;54:1230-9. doi:10.1093/cid/cis199.

5. Walkty A. Cardiobacterium hominis endocarditis: A case report and review of the literature. Can J Infect Dis Med Microbiol 2005;16:293-7.

6. Petti CA, Bhally HS, Weinstein MP, et al. Utility of extended blood culture incubation for isolation of haemophilus, and kingella organisms. J Clin Microbiol 2006;44:1-4. doi:10.1128/JCM.44.1.257.

7. Baddour LM, Wilson WR, Bayer AS, et al. Infective endocarditis in adults: Diagnosis, antimicrobial therapy, and management of complications: A scientific statement for healthcare professionals from the American Heart Association. Circulation 2015. doi:10.1161/CIR.0000000000000296.

8. Malani AN, Aronoff DM, Bradley SF, Kauffman CA. Cardiobacterium hominis endocarditis: Two cases and a review of the literature. Eur J Clin Microbiol Infect Dis 2006;25:587-95. doi:10.1007/s10096-006-0189-9.

9. Public Health England. Identification of haemophilus species and the HACEK group of organisms. UK Stand Microbiol Investig 2012;12:1-18.

10. Okell C, Elliott S. Bacteraemia and oral sepsis: with special reference to the aetiologu of subacute endocarditis. Lancet 1935;226:869-72.

11. Wilson W, Taubert KA, Gewitz M, et al. Prevention of infective endocarditis: Guidelines from the American Heart Association. Circulation 2007;116:1736-54. doi:10.1161/CIRCULATIONAHA.106.183095.

12. Song JK. Infective endocarditis involving an apparently structurally normal valve: New epidemiological trend? Korean J Intern Med 2015;30:434-42. doi:10.3904/kjim.2015.30.4.434.

13. Habib G, Lancellotti P, Antunes MJ, et al. 2015 ESC Guidelines for the management of infective endocarditis. Eur Heart J 2015;36. doi:10.1093/ eurheartj/ehv319.

14. Van der Meer J, van Wijk W, Thompson J, et al. Efficacy of antibiotic prophylaxis for prevention of native-valve endocarditis. Lancet 1992;339:135-9.

15. Glenny A, Oliver R, Roberts GJ, Hooper L, Worthington HV. Antibiotics for the prophylaxis of bacterial endocarditis in dentistry (Review). Cochrane Database Syst Rev 2013. doi:10.1002/14651858.CD003813.pub4.

16. Dayer MJ, Jones S, Prendergast B, et al. Incidence of infective endocarditis in England, 2000-13: A secular trend, interrupted time-series analysis. Lancet 2015;385:1219-28. doi:10.1016/S0140-6736(14)62007-9.

17. National Institute for Health and Care Excellence. Prophylaxis against infective endocarditis. Secondary prophylaxis against infective endocarditis. 2016. Available at: https://www.nice.org.uk/guidance/cg64.

18. Thornhill MH, Dayer MJ, Prendergast B, et al. Incidence and nature of adverse reactions to antibiotics used as endocarditis prophylaxis. J Antimicrob Chemother 2015;70:2382-8. doi:10.1093/jac/dkv115. 